\title{
MORFOLOGIA FOLIAR DE INDIVÍDUOS JOVENS E ADULTOS DE Caesalpinia echinata LAM. NUMA FLORESTA SEMIDECÍDUA DO SUL DA BAHIA ${ }^{1}$
}

\author{
Maria Cristina Sanches², Marcelo Schramm Mielke², Clara Sampaio Dias de Souza ${ }^{4}$, Antonio José \\ Dias Vieira ${ }^{5}$, Márdel Miranda Mendes Lopes ${ }^{6}$ e Milton Barbosa da Silva Júnior ${ }^{7}$
}

\begin{abstract}
RESUMO - Caesalpinia echinata Lam. (Caesalpiniaceae) é uma espécie arbórea que ocorre naturalmente no interior da floresta e atinge os estratos superiores do dossel por meio de pequenas clareiras. Objetivou-se, neste estudo, comparar alguns aspectos da morfologia foliar entre indivíduos adultos, cujas folhas se encontravam em pleno sol e jovens no sub-bosque. O estudo foi realizado numa floresta semidecídua, localizada no Município de Jussari, Bahia. Inicialmente, foram localizados e marcados oito indivíduos adultos e oito jovens para cada adulto. Foram coletadas e analisadas oito folhas para cada adulto e três para cada jovem, em duas épocas (setembro/ novembro de 2004 e abril de 2005). Em geral, as áreas das folhas, das ráquis e dos folíolos, o comprimento das ráquis, a largura das folhas, o número de pinas e a massa seca das folhas foram significativamente superiores nos indivíduos jovens, ao passo que as massas específicas das folhas e dos folíolos foram significativamente superiores nos indivíduos adultos. Tanto nos adultos quanto nos jovens, nas duas épocas de coleta foram verificadas relações altamente significativas entre a massa seca e a área das folhas. Os resultados indicaram que folhas de C. echinata apresentam características que maximizam a absorção de luz onde este recurso é limitante e, ao mesmo tempo, direcionam maior alocação de carbono para os tecidos de suporte. Tais resultados estão de acordo com o observado no estádio sucessional da espécie analisada e com a sua estratégia de ocupação dos espaços gerados pela formação de pequenas clareiras, em ambiente de mata semidecídua.
\end{abstract}

Palavras-chave: Área foliar, mata Atlântica e regeneração natural.

\section{LEAF MORPHOLOGY OF SAPLINGS AND ADULT INDIVIDUALS OF Caesalpinia echinata LAM. IN A SEMIDECIDUAL FOREST OF SOUTHERN BAHIA, BRAZIL}

\begin{abstract}
Caesalpinia echinata Lam. (Caesalpiniaceae) is a tree species that naturally regenerates in the forest understory and reaches the upper canopy through small gaps. We conducted a study with the objective of comparing some aspects of leaf morphology of adult individuals, in which the leaves were exposed to full sunlight, and saplings, grown in the forest understory. The study was conducted in a semidecidual forest in Jussari, southern Bahia, Brazil. Initially, eight adult and eight sapling individuals were selected and marked. In September/October, 2004 and April, 2005 eight leaves for each adult and three leaves for each sapling individual were collected and analyzed. In general, the area of leaves, rachis and leaflets, the length of rachis, the width of leaves, the number of pinnes and the dried mass of leaves were significantly higher in young individuals. Notwithstanding, the specific mass of leaves and leaflets were significantly higher in the adults. For both, adults and saplings, strong relationships were observed between the leaf dried mass and leaf area. Based on the results, we conclude that the leaves of C. echinata present characteristics that allow the maximization
\end{abstract}

\footnotetext{
${ }^{1}$ Recebido em 05-12-2007 e aceito para publicação em 23.06.2009.

${ }^{2}$ Universidade Federal de Uberlândia, Instituto de Biologia, Campus Umuarama Bloco 2D, Uberlândia, MG- Brasil. E-mail: <sanchesmc@inbio.ufu.br>.

${ }^{3}$ Universidade Estadual de Santa Cruz, Departamento de Ciências Biológicas Ilheus, BA - Brasil. E-mail: <msmielke@uesc.br>. ${ }^{4}$ Universidade Estadual de Campinas, Instituto de Biologia, Departamento de Botânica, Campinas, SP - Brasil. E-mail: <clarasds@yahoo.com.br>.

${ }^{5}$ Instituto Federal de Educação, Ciência e Tecnologia do Maranhão,ZZe Doca, MA-Brasil. E-mail:<antonio_vieira_pa@yahoo.com.br>. ${ }^{6}$ Universidade de São Paulo, Instituto de Biociências. Sao Paulo, SP - Brasil. E-mail:<mardelmml@yahoo.com.br>.

${ }^{7}$ Universidade Federal de Minas Gerais, Instituto de Ciências Biológicas, Departamento de Biologia Geral, Belo Horizonte MG - Brasil. E-mail: <miltonbsjunior@yahoo.com.br>.
} 
of light absorption in environments in which light radiation is a limiting factor, at the same time allocating carbon to the support tissues. The results obtained in the present study were in accordance with the successional status of this tree species and its strategies to occupy small canopy gaps of the semidecidual forest environments.

Keywords: Atlantic Forest, leaf area and natural regeneration.

\section{INTRODUÇÃO}

A folha é o órgão mais importante para a realização das trocas de carbono e água entre planta e atmosfera. De acordo com as características do ambiente em que se encontram, as folhas apresentam adaptações anatômicas, morfológicas e fisiológicas que permitem a manutenção de um balanço positivo de carbono. Folhas de sombra são, em geral, mais finas, apresentam menor massa específica e menor razão entre parênquimas paliçádico e lacunoso do que folhas de sol (ARANDA et al., 2004). Tais características podem variar entre grupos ecológicos ou entre espécies, e influenciam a capacidade de sobreviver e crescer em ambientes de clareiras ou no sub-bosque das matas fechadas. Assim, a avaliação das mudanças na morfologia foliar em resposta a diferentes ambientes de crescimento proporciona critérios importantes para analisar a plasticidade morfológica e fisiológica com relação às adaptações ao sol ou sombra, bem como para prover informações referentes à compreensão dos aspectos ecofisiológicos relacionados com a regeneração natural e a reintrodução de espécies arbóreas nativas no seu habitat.

O pau-brasil (Caesalpinia echinata Lam. Leguminosae Caesalpinioideae) é uma espécie arbórea nativa, de importâncias econômica, ambiental e cultural para a região Sul do Estado da Bahia. Podendo atingir até $30 \mathrm{~m}$ de altura e $1 \mathrm{~m}$ de diâmetro; ocorre do Ceará ao Rio de Janeiro, sendo frequente no Sul da Bahia, ocorrendo desde a Floresta Estacional à Floresta Ombrófila (CARVALHO, 1994). É uma espécie clímax, que atinge o dossel após a formação de pequenas clareiras. O pau-brasil vem sendo explorado extensivamente desde o início da colonização europeia e atualmente encontra-se ameaçado de extinção; sendo poucos os locais onde ainda possam ser encontradas populações nativas. Apesar de ser espécie relativamente bem estudada do ponto de vista de seus caracteres taxonômicos, fitoquímicos e de propagação (CORRÊA, 2003; REZENDE et al., 2004; AGUIAR et al., 2005; MELO et al., 2007), são poucos os estudos realizados sobre a ecofisiologia dessa espécie, particularmente em florestas nativas, utilizando-se plantas oriundas de regeneração natural (GEBLER et al., 2005). Tais estudos podem no futuro proporcionar subsídios para a compreensão dos aspectos relacionados à regeneração natural dessa espécie numa floresta semidecídua do Sul da Bahia. Considerando a importância da C. echinata do ponto de vista ecológico e comercial, este estudo foi realizado com o objetivo de comparar aspectos da morfologia foliar entre plantas adultas, cujas folhas se encontravam em pleno sol e os indivíduos jovens, no sub-bosque.

\section{MATERIAL E MÉTODOS}

O estudo foi realizado na Reserva Particular do Patrimônio Natural (RPPN) Serra do Teimoso, em Jussari, Bahia (http://www.serradoteimoso.com.br), nas coordenadas geográficas $15^{\circ} 09^{\prime} 16,2^{\prime \prime}$ S e 39³1'25,1' W. De acordo com a classificação de Köppen, o clima da região de Jussari varia de úmido a sub úmido e de seco a sub úmido. A temperatura média anual é de 23,5 ${ }^{\circ} \mathrm{C}$. O período chuvoso estende-se de janeiro a março, com os índices pluviométricos variando de 1.500 a 1.800 $\mathrm{mm}$ anuais, e o período seco, entre junho e dezembro. A reserva localiza-se na transição entre a floresta úmida do litoral e a floresta mais seca do interior, sendo formada por uma mata de encosta (AMORIM et al., 2005).

Em agosto de 2004, oito árvores adultas foram localizadas e marcadas. Ao redor de cada indivíduo adulto foram também localizados e marcados oito jovens, selecionando-se sempre aqueles que estavam mais próximos de cada um dos adultos. Os indivíduos adultos foram divididos em grupos com letras maiúsculas correspondentes às árvores $(\mathrm{A}, \ldots, \mathrm{H})$ e números correspondentes aos jovens $(1, \ldots, 8)$. Foram medidos, em cada um dos indivíduos jovens, a altura total e da primeira ramificação, o número de ramos, a distância da árvore adulta e o diâmetro. Como se tratava de plantas de baixa estatura, o diâmetro foi medido logo abaixo da primeira ramificação e, naquelas com ramificações muito próximas ao solo, o diâmetro foi medido nos dois ramos principais abaixo das ramificações secundárias, 
sendo posteriormente calculada a média das duas medições. Nos indivíduos com mais de $2 \mathrm{~m}$ de altura e nas árvores, o diâmetro foi medido à altura do peito.

No mês de setembro (árvores C, D, E e F) e novembro (árvores A, B, G e H) de 2004, oito folhas de cada uma das plantas adultas e três de cada uma das plantas jovens foram coletadas. Após a coleta, a área das folhas (AF) foi medida utilizando-se um medidor automático de área foliar Li-3100 (Li-Cor, USA). Em seguida, todas as folhas foram fotocopiadas e colocadas em estufa, à temperatura média de $70^{\circ} \mathrm{C}$, até a obtenção de massa seca constante (MSF). A partir das fotocópias, foram medidos o comprimento da ráquis (CR), a largura das folhas (LF) e o número de pinas (NP). A partir de MSF e de AF, foi calculada a massa específica das folhas $(\mathrm{MEF}=\mathrm{MSF} / \mathrm{AF})$.

Em abril de 2005, durante a realização do III Curso de Campo de Ecologia de Dossel (http:// www.ib.unicamp.br/profs/fsantos/ne313/index), novamente foram coletados dados em oito folhas de cada uma das árvores C, D, E e F, e três de cada um dos respectivos indivíduos jovens. Logo após a coleta, as folhas e suas ráquis foram escaneadas e as áreas das folhas $(\mathrm{AF})$ e da ráquis ( $\mathrm{AR}$ ), calculadas por meio do software ImageJ 1.31v (http://rsb.info.nih.gov/ij/), sendo a área dos folíolos (AFL) calculada subtraindo-se AF de AR. Após esse procedimento, as folhas foram colocadas num recipiente de plástico hermeticamente fechado, com a base dos pecíolos imersa em água durante 24 horas, para obtenção da massa túrgida da folha (MTF), da ráquis (MTR) e dos folíolos (MTFL). Em seguida, as partes de cada folha foram colocadas em estufa à temperatura média de $70{ }^{\circ} \mathrm{C}$ até a obtenção de massa seca constante de ráquis (MSR) e folíolos (MSFL), sendo a massa seca das folhas (MSF) calculada pela soma entre MSR e MSFL. Logo em seguida, foi calculada a massa específica de folhas (MEF) e de folíolos (MEFL), dividindo-se MSF por AF e MSFL por AFL, respectivamente. $\mathrm{O}$ teor máximo de água das folhas (TMAF), folíolos (TMAFL) e ráquis (TMAR) foram calculados pela seguinte fórmula: TMA $=[(\mathrm{MT}-\mathrm{MS}) /$ MT]x100, em que MT = massa túrgida e MS = massa seca (g). As razões de massa de folíolos (RMFL) e de ráquis ( $R M R$ ) foram calculadas dividindo-se as respectivas massas secas dos folíolos e da ráquis pela massa seca das folhas inteiras. Foram também medidas as densidades de fluxo de fótons fotossinteticamente ativos (DFF), por meio de um sensor quântico BQM-
SUN (Apogee Instruments Inc, USA) e de um sensor linear da Licor LI-191SA (Li-Cor, USA) acoplado a um Datalogger LI-1000, nos diferentes pontos de coleta. Foram efetuadas medidas de DFF no mesmo instante, a pleno sol e no interior da floresta, entre $9 \mathrm{~h} 30$ e $15 \mathrm{~h}$, em dia de céu claro. No interior da floresta, as medições de DFF foram realizadas o mais próximo possível das folhas das plantas jovens.

Para a análise das variáveis morfológicas, foi adotado o delineamento experimental inteiramente casualizado com dois tratamentos e oito repetições, com uma unidade experimental para adultos e três para jovens. Os resultados foram analisados mediante análise de variância, independentemente de cada indivíduo adulto e indivíduos jovens. Relações entre variáveis dimensionais e estruturais e entre os diferentes componentes das folhas foram analisadas mediante regressões lineares, separando-se indivíduos adultos e jovens.

\section{RESULTADOS}

O diâmetro dos indivíduos adultos variou entre 172 e 615 mm, enquanto o diâmetro médio dos indivíduos jovens oscilou entre 4 e $10 \mathrm{~mm}$ (Tabela 1). As médias de altura (ALT) dos indivíduos jovens variaram entre 1,3 e 1,9 m, e as médias de número de ramos (NR) entre 3,3 e 6,6. Em geral, foi possível encontrar número relativamente grande de indivíduos jovens a uma distância inferior que $10 \mathrm{~m}$ da árvore adulta, e as médias de distância da árvore adulta (DAA) variaram entre 3,9 e 7,6 m.

Na Figura 1, pode ser visto o aspecto geral das folhas de C. echinata, sendo representada por um indivíduo jovem, cuja folha se desenvolveu no sub-bosque, e pela folha de um adulto que se formou no dossel superior. As condições microclimáticas distintas entre o dossel superior e o sub-bosque afetaram várias características morfológicas analisadas. Os valores médios encontrados para MSF, AF, CR e NP foram, em geral, maiores nos indivíduos jovens do que nos adultos, ao passo que MEF foi maior nos indivíduos adultos (Tabela 2). Os valores calculados para MEF refletem a relação entre a área menor das folhas das árvores e a massa seca delas, uma vez que as diferenças percentais médias entre os valores obtidos para MSF e AF nos indivíduos adultos e jovens foram de 168 e $232 \%$, respectivamente. As maiores diferenças percentuais entre as médias obtidas dos indivíduos adultos e jovens foram encontradas nas variáveis

R. Árvore, Viçosa-MG, v.33, n.5, p.885-893, 2009 
Tabela 1 -Diâmetros das oito árvores adultas de Caesalpinia echinata, seguidos dos valores médios ( \pm dp) do diâmetro (D), da altura (ALT), do número de ramos (NR) e da distância da árvore adulta (DAA) dos respectivos indivíduos jovens associados, numa mata semidecídua, em Jussari, Bahia, Brasil $(\mathrm{n}=8)$.

Table 1 - Diameter of eight adult trees of Caesalpinia echinata, and diameter (D), height (ALT), branch numbers (NR) and distance from adult tree of saplings associations in a semi-deciduous tropical forest, Jussari Bahia, Brazil $(n=8)$. The data from saplings represent mean $( \pm d p)$.

\begin{tabular}{cccccc}
\hline Árvore & $\begin{array}{c}\text { Diâmetro do } \\
\text { adulto }(\mathrm{mm})\end{array}$ & D $(\mathrm{mm})$ & ALT $(\mathrm{m})$ & Indivíduos jovens & DAA (m) \\
\hline A & 238 & $10 \pm 6$ & $1,5 \pm 0,7$ & $4,1 \pm 1,7$ & $7,6 \pm 2,7$ \\
B & 524 & $10 \pm 3$ & $1,5 \pm 0,7$ & $6,6 \pm 2,0$ & $5,8 \pm 2,7$ \\
C & 608 & $4 \pm 1$ & $1,3 \pm 0,4$ & $5,8 \pm 2,8$ & $7,1 \pm 1,8$ \\
D & 368 & $5 \pm 3$ & $1,6 \pm 0,8$ & $4,9 \pm 2,7$ & $4,5 \pm 1,8$ \\
F & 477 & $5 \pm 2$ & $1,5 \pm 0,7$ & $6,1 \pm 2,7$ & $4,8 \pm 1,7$ \\
G & 615 & $6 \pm 4$ & $1,9 \pm 1,1$ & $4,8 \pm 2,1$ & $5,2 \pm 3,8$ \\
H & 533 & $7 \pm 2$ & $1,3 \pm 0,7$ & $4,5 \pm 3,2$ & $3,9 \pm 1,7$ \\
I & 172 & $9 \pm 4$ & $1,4 \pm 1,3$ & $3,3 \pm 1,3$ & $4,5 \pm 1,1$ \\
\hline
\end{tabular}

Tabela 2-Massa seca (MSF), área foliar (AF), massa específica (MEF), comprimento da ráquis (CR), largura (LF) e número de pinas (NP) em folhas de árvores adultas $(\mathrm{A}, \ldots \mathrm{H})$ e jovens $(\mathrm{AJ}, \ldots \mathrm{HJ})$ de Caesalpinia echinata, numa mata semidecídua, Jussari, Bahia, Brasil. Setembro e outubro de 2004 ( $(n=8 \pm$ dp). Comparações de médias entre indivíduos adultos e jovens realizada pela análise da variância. ** $p<0,01, * p<0,05 \mathrm{e}^{\mathrm{ns}} p \geq 0,05$.

Table 2 - Dry mass $(M S F)$, leaf area (AF), specific mass $(M E F)$, rachis length, width and pinnae numbers in leaves from adult $(A, \ldots H)$ and sapling $(A J, \ldots H J)$ trees of Caesalpinia echinata from a semi-deciduous tropical forest, Jussari, Bahia, Brazil. The data were collected in September and October 2004 and represent the mean $\pm d p ; n=8$. Comparisons of the means of adult trees and saplings were performed by the ANOVA. ** $p<0,01, * p<0,05$ and $n s p \geq 0,05$.

\begin{tabular}{|c|c|c|c|c|c|c|}
\hline Árvore & $\operatorname{MSF}(\mathrm{g})$ & $\mathrm{AF}\left(\mathrm{cm}^{2}\right)$ & $\operatorname{MEF}\left(\mathrm{g} \mathrm{m}^{-2}\right)$ & $\mathrm{CR}(\mathrm{cm})$ & $\mathrm{LF}(\mathrm{cm})$ & NP \\
\hline $\mathrm{A}$ & $0,4 \pm 0,2 * *$ & $46,1 \pm 14,5^{* *}$ & $90,0 \pm 8,2 * *$ & $5,9 \pm 1,1$ *** & $11,4 \pm 1,5^{* *}$ & $6,8 \pm 0,9^{* *}$ \\
\hline $\mathrm{AJ}$ & $0,7 \pm 0,2$ & $121,9 \pm 21,3$ & $57,8 \pm 5,7$ & $13,2 \pm 1,7$ & $16,4 \pm 1,1$ & $9,4 \pm 0,8$ \\
\hline$\Delta \%$ & 171 & 265 & 64 & 223 & 144 & 140 \\
\hline B & $0,4 \pm 0,1^{* *}$ & $53,9 \pm 14,5 * *$ & $72,0 \pm 11,3 *$ & $5,5 \pm 0,9 * *$ & $10,2 \pm 1,4 * *$ & $7,0 \pm 0,8^{*}$ \\
\hline $\mathrm{BJ}$ & $0,9 \pm 0,3$ & $148,4 \pm 39,1$ & $59,7 \pm 7,3$ & $12,8 \pm 2,0$ & $15,1 \pm 1,7$ & $10,2 \pm 1,0$ \\
\hline$\Delta \%$ & 226 & 276 & 83 & 234 & 149 & 146 \\
\hline $\mathrm{C}$ & $0,8 \pm 0,1 * *$ & $79,7 \pm 15,4 * *$ & $99,0 \pm 19,5^{* *}$ & $5,3 \pm 1,5^{* *}$ & $12,0 \pm 2,5^{* *}$ & $7,5 \pm 1,6^{*}$ \\
\hline $\mathrm{CJ}$ & $1,1 \pm 0,2$ & $171,4 \pm 30,5$ & $62,8 \pm 5,7$ & $12,1 \pm 1,4$ & $16,5 \pm 2,3$ & $9,3 \pm 1,1$ \\
\hline$\Delta \%$ & 141 & 215 & 63 & 228 & 138 & 124 \\
\hline $\mathrm{D}$ & $0,6 \pm 0,2^{\mathrm{ns}}$ & $66,4 \pm 28,1 * *$ & $91,5 \pm 19,1^{* *}$ & $6,7 \pm 1,4^{* *}$ & $13,1 \pm 2,4^{\mathrm{ns}}$ & $7,4 \pm 1,2^{* *}$ \\
\hline DJ & $0,7 \pm 0,3$ & $122,5 \pm 39,8$ & $55,7 \pm 6,7$ & $11,8 \pm 2,0$ & $14,8 \pm 1,8$ & $9,3 \pm 1,3$ \\
\hline$\Delta \%$ & 119 & 185 & 61 & 177 & 113 & 127 \\
\hline $\mathrm{E}$ & $0,4 \pm 0,1 * *$ & $51,8 \pm 15,2 * *$ & $78,6 \pm 8,4^{* *}$ & $5,8 \pm 1,3^{* *}$ & $11,6 \pm 2,7 * *$ & $6,5 \pm 1,3^{* *}$ \\
\hline EJ & $1,1 \pm 0,3$ & $172,5 \pm 44,7$ & $63,4 \pm 8,6$ & $12,4 \pm 2,6$ & $17,0 \pm 1,8$ & $9,1 \pm 0,9$ \\
\hline$\Delta \%$ & 273 & 333 & 81 & 212 & 147 & 140 \\
\hline $\mathrm{F}$ & $0,8 \pm 0,1^{\mathrm{ns}}$ & $71,9 \pm 12,5 * *$ & $104,9 \pm 14,1 * *$ & $5,7 \pm 0,8 * *$ & $12,3 \pm 1,3 * *$ & $6,8 \pm 1,6^{*}$ \\
\hline FJ & $0,7 \pm 0,2$ & $124,2 \pm 27,3$ & $56,2 \pm 7,6$ & $11,8 \pm 2,6$ & $15,4 \pm 1,4$ & $8,8 \pm 1,4$ \\
\hline$\Delta \%$ & 94 & 173 & 54 & 208 & 126 & 130 \\
\hline $\mathrm{G}$ & $0,5 \pm 0,2^{* *}$ & $64,7 \pm 25,8^{* *}$ & $74,2 \pm 4,3^{* *}$ & $5,0 \pm 1,0^{* *}$ & $11,5 \pm 2,3 * *$ & $6,8 \pm 1,2^{* *}$ \\
\hline GJ & $1,1 \pm 0,2$ & $177,8 \pm 41,2$ & $60,2 \pm 4,9$ & $14,7 \pm 1,8$ & $16,8 \pm 1,6$ & $9,4 \pm 0,8$ \\
\hline$\Delta \%$ & 221 & 275 & 81 & 293 & 146 & 139 \\
\hline $\mathrm{H}$ & $0,8 \pm 0,3^{\mathrm{ns}}$ & $102,4 \pm 31,7 *$ & $78,9 \pm 13,0 * *$ & $7,7 \pm 1,0 * *$ & $16,1 \pm 2,5^{\mathrm{ns}}$ & $7,1 \pm 1,0 * *$ \\
\hline $\mathrm{HJ}$ & $0,8 \pm 0,2$ & $138,7 \pm 23,3$ & $58,8 \pm 6,6$ & $14,5 \pm 1,7$ & $15,7 \pm 1,6$ & $9,9 \pm 1,3$ \\
\hline$\Delta \%$ & 100 & 135 & 74 & 187 & 98 & 139 \\
\hline
\end{tabular}

R. Árvore, Viçosa-MG, v.33, n.5, p.885-893, 2009 

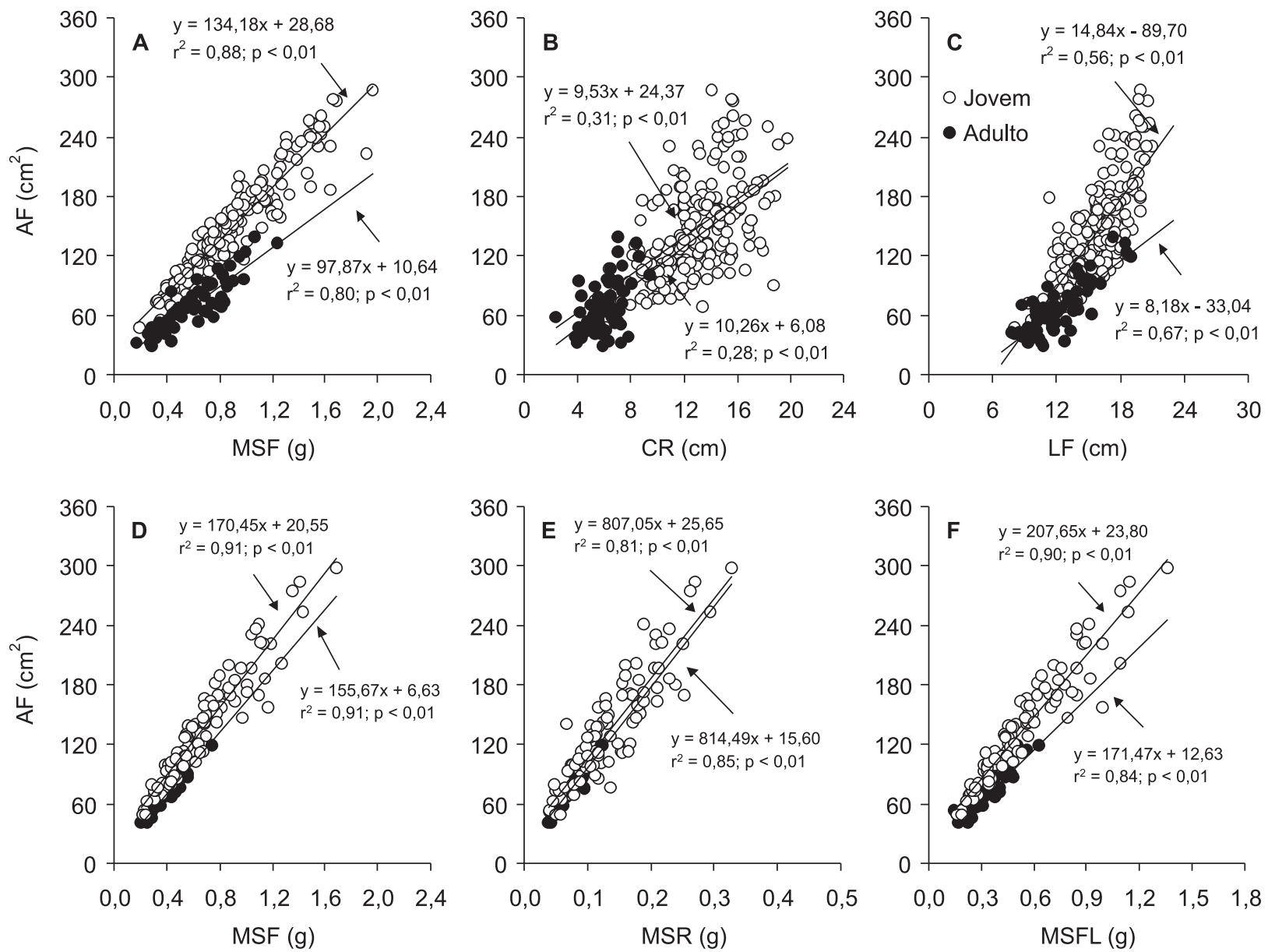

Figura 2 - Relações entre a área foliar (AF) e a massa seca das folhas (MSF) (A, D), o comprimento da ráquis (CR) (B), a largura das folhas (LF) (C) e a massa seca da ráquis (MSR) (E) e dos folíolos (MSFL) (F) em indivíduos jovens e adultos de Caesalpinia echinata, numa mata semidecídua, em Jussari, Bahia, Brasil. A, B e C = observações em setembro e outubro de 2004; D, E e F observações em abril de 2005. Cada ponto representa uma medição.

Figure 2 - Relations between the leaf area (AF) and leaf dry mass (MSF) (A, D), rachis length $(C R)(B)$, leaf width (LF) $(C)$ and dry mass rachis $(M S R)$ and leaflet $(M S F L)(F)$ for both adult tree and saplings of Caesalpinia echinata from a semi-deciduous tropical forest, Jussari, Bahia, Brazil. The data from A, B, C, represent the data collected in September and October 2004 and D, E, F represent the data collected in April 2005. Each point is one measure.

AF e CR, em que praticamente em todos os conjuntos analisados foram encontradas diferenças altamente significativas $(p<0,01)$ entre adultos e jovens.

Relações entre MS e AF indicaram elevados valores para os coeficientes de determinação $\left(r^{2}\right)$ (Figura 2AD). Relações lineares entre CR e AF e entre LF e AF, no entanto apresentaram baixos valores de $\mathrm{r}^{2}$ (Figura 2B2C); embora a relação entre LF e AF tenha apresentado tendência bastante semelhante àquela encontrada na relação entre MS e AF (Figura 2AC). Da mesma forma como foi observado nas relações entre MS e AF, verificou-se a tendência de os valores de AF serem menores nas folhas das plantas adultas do que nas folhas das plantas jovens para um mesmo valor de $\mathrm{CR}$. As relações entre massa seca das folhas, das ráquis e dos folíolos e as respectivas áreas indicaram elevados valores de $r^{2}$ (Figura 2EF).

Assim como ocorreu nas folhas coletadas em setembro e novembro de 2004, os valores médios encontrados na coleta de 2005 para AF e AR foram, em geral, maiores nos indivíduos jovens do que nos 

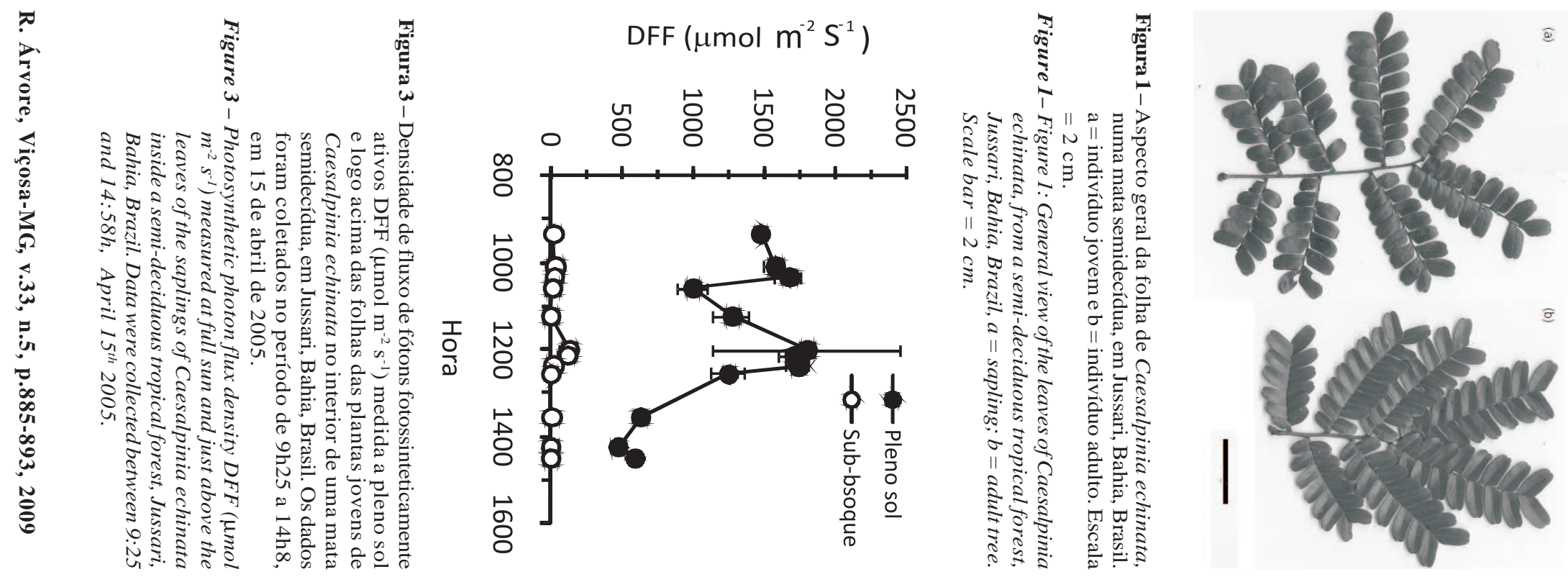

Tabela 3-Áreas de folhas (AF), das ráquis (AR) e dos folíolos (AFL), massa específica de folhas (MEF) e de folíolos (MEFL), teor máximo de água nas folhas (TMAF), na ráquis (TMAR) e nos folíolos (TMAF) e razões de massa de ráquis (RMR) e de folíolos (RMFL) de árvores adultas (C ....F) e jovens (CJ,...FJ) de Caesalpinia echinata, numa mata semidecídua, em Jussari, Bahia, Brasil. Abril de 2005 ( $\mathrm{n}=8 \pm$ dp). Comparação de medias entre indivíduos adultos e jovens realizada pela análise da variância. $* * p<0,01, * p<0,05 \mathrm{e}^{\mathrm{ns}} p \geq 0,05$.

Table 3- Leaf, rachis and leaflet area, specific leaf mass (MEF) and specific leaflet mass (MEFL), maximum water content in leaves (TMAF), in rachis $(T M A R)$ and in leaflet (TMAF) and rachis mass ratio $(R M R)$ and leaflet mass ratio $(R M F L)$ from adult trees $(C, \ldots F)$ and saplings $(C J, \ldots F J)$ of Caesalpinia echinata from a semi-deciduous tropical forest, Jussari, Bahia, Brazil. The data were collected in April 2005 and represent the mean $\pm \mathrm{dp} ; \mathrm{n}=8$. Comparisons of the means of adult tree and saplings were performed by the ANOVA. **p<0,01,*p<0,05 and ${ }^{n s} p \geq$.

\begin{tabular}{|c|c|c|c|c|c|c|c|c|c|c|}
\hline Árvore & $A F\left(\mathrm{~cm}^{2}\right)$ & $\mathrm{AR}\left(\mathrm{cm}^{2}\right)$ & $\operatorname{AFL}\left(\mathrm{cm}^{2}\right)$ & $\operatorname{MEF}\left(\mathrm{g} \mathrm{m}^{-2}\right)$ & $\operatorname{MEFL}\left(\mathrm{g} \mathrm{m}^{-2}\right)$ & TMAF (\%) & TMAR (\%) & TMAFL (\%) & $\operatorname{RMR}\left(\mathrm{g} \mathrm{g}^{-1}\right)$ & RMFL $\left(\mathrm{g} \mathrm{g}^{-1}\right)$ \\
\hline $\mathrm{C}$ & $71,3 \pm 18,5^{*}$ & $4,4 \pm 1,3 \mathrm{~ns}$ & $66,9 \pm 18,3^{*}$ & $57,6 \pm 5,8^{* *}$ & $48,8 \pm 9,1^{*}$ & $65,3 \pm 3,1 \mathrm{~ns}$ & $59,6 \pm 7,3 \mathrm{~ns}$ & $63,9 \pm 6,8 \mathrm{~ns}$ & $0,21 \pm 0,11^{\text {ns }}$ & $0,79 \pm 0,11^{\mathrm{ns}}$ \\
\hline CJ & $106,3 \pm 36,8$ & $5,6 \pm 1,7$ & $100,6 \pm 35,5$ & $47,0 \pm 6,0$ & $39,9 \pm 5,8$ & $66,6 \pm 1,3$ & $62,2 \pm 2,1$ & $63,4 \pm 3,2$ & $0,19 \pm 0,03$ & $0,80 \pm 0,03$ \\
\hline$\Delta \%$ & 149 & 128 & 150 & 82 & 82 & 102 & 104 & 99 & 93 & 102 \\
\hline $\mathrm{D}$ & $66,5 \pm 22,9^{* *}$ & $3,3 \pm 1,1 * *$ & $63,2 \pm 21,8^{* *}$ & $60,8 \pm 3,5^{* *}$ & $55,0 \pm 3,4 * *$ & $63,7 \pm 1,5^{*}$ & $57,8 \pm 6,9 \mathrm{~ns}$ & $58,4 \pm 3,3^{*}$ & $0,14 \pm 0,01 * *$ & $0,86 \pm 0,01^{* *}$ \\
\hline DJ & $163,1 \pm 48,5$ & $8,1 \pm 2,1$ & $155,0 \pm 46,4$ & $48,4 \pm 4,3$ & $41,0 \pm 3,5$ & $65,6 \pm 1,8$ & $62,1 \pm 1,4$ & $61,8 \pm 2,5$ & $0,19 \pm 0,02$ & $0,81 \pm 0,02$ \\
\hline$\Delta \%$ & 245 & 245 & 245 & 80 & 75 & 103 & 107 & 106 & 139 & 94 \\
\hline $\mathrm{E}$ & $79,7 \pm 20,3 *$ & $4,8 \pm 1,1^{*}$ & $74,9 \pm 19,3^{*}$ & $59,9 \pm 3,6^{* *}$ & $53,2 \pm 3,8 * *$ & $66,7 \pm 1,6 \mathrm{~ns}$ & $65,7 \pm 1,4 \mathrm{~ns}$ & $59,2 \pm 10,8 \mathrm{~ns}$ & $0,16 \pm 0,02 * *$ & $0,84 \pm 0,02 * *$ \\
\hline EJ & $122,1 \pm 36,2$ & $6,9 \pm 2,1$ & $115,1 \pm 34,3$ & $46,8 \pm 4,0$ & $38,6 \pm 3,5$ & $66,8 \pm 2,3$ & $62,9 \pm 3,6$ & $64,0 \pm 2,9$ & $0,22 \pm 0,03$ & $0,78 \pm 0,03$ \\
\hline$\Delta \%$ & 153 & 145 & 154 & 78 & 73 & 100 & 96 & 108 & 134 & 93 \\
\hline $\mathrm{F}$ & $80,8 \pm 19,7 * *$ & $4,9 \pm 1,1 * *$ & $75,9 \pm 18,6^{* *}$ & $55,8 \pm 5,8^{\text {ns }}$ & $48,1 \pm 5,6^{\text {ns }}$ & $63,7 \pm 2,3 \mathrm{~ns}$ & $59,8 \pm 4,9 \mathrm{~ns}$ & $60,3 \pm 2,8 \mathrm{~ns}$ & $0,19 \pm 0,02 *$ & $0,81 \pm 0,02 *$ \\
\hline FJ & $148,3 \pm 41,2$ & $7,6 \pm 1,7$ & $140,6 \pm 39,6$ & $54,3 \pm 4,4$ & $45,5 \pm 4,0$ & $64,4 \pm 1,6$ & $63,1 \pm 2,5$ & $61,1 \pm 2,2$ & $0,21 \pm 0,01$ & $0,79 \pm 0,01$ \\
\hline$\Delta \%$ & 184 & 155 & 185 & 97 & 94 & 101 & 106 & 101 & 109 & 98 \\
\hline
\end{tabular}


adultos, ao passo que MEF e MEFL foram maiores nos indivíduos adultos (Tabela 3). As maiores diferenças percentuais entre as médias obtidas dos indivíduos adultos e jovens foram encontradas nas variáveis AF, AR e AFL, em que para praticamente todos os conjuntos analisados, foram encontradas diferenças altamente significativas $(p<0,01)$. Já os valores médios de TMAF, TMAR e TMAFL não indicaram, em termos gerais, diferenças significativas entre adultos e jovens, embora tenha sido verificada tendência de maiores valores de teor máximo de água nas folhas dos indivíduos jovens (Tabela 3). No entanto, folhas de indivíduos jovens apresentaram, de modo geral, maiores valores de RMR e menores valores de RMFL quando comparados com as folhas de indivíduos adultos.

Os valores de DFF no sub-bosque foram, em média, de somente $3 \%$ em relação aos valores medidos em pleno sol (Figura 3). Os valores médios de DFF em pleno sol variaram entre 482 e $1.809 \mu \mathrm{mol}$ fótons $\mathrm{m}^{-2} \mathrm{~s}^{-1}$, ao passo que, no sub-bosque, tais valores estiveram entre $4 \mathrm{e}$ $133 \mu \mathrm{mol}$ fótons $\mathrm{m}^{-2} \mathrm{~s}^{-1}$.

\section{DISCUSSÃO}

Caesalpinia echinata é uma espécie arbórea que pode atingir até $30 \mathrm{~m}$ de altura e $1 \mathrm{~m}$ de diâmetro, sendo capaz de se regenerar no sub-bosque das florestas tropicais. Diferenças no comprimento da ráquis e no número de pinas foram detectadas entre os indivíduos adultos e jovens dessa espécie. O maior comprimento da ráquis nos indivíduos jovens pode estar indicando a influência da baixa razão vermelho/vermelho distante que predomina no sub-bosque (LEE et al., 1997). O aumento em extensão de caule e, ou, pecíolo tem sido considerado típica resposta morfogênica que ocorre em condições de sombra natural (APHALO e BALLARÉ, 1995), resultando em maior probabilidade de captura de radiação luminosa.

As mudanças decorrentes do aumento da DFF podem resultar na diminuição da área foliar e no aumento da sua espessura e da sua densidade estomática (DUZ et al., 2004; LIMA et al., 2006). Essa tendência foi confirmada neste estudo, em que as folhas dos indivíduos adultos, cujas folhas estavam expostas a altas irradiâncias (Tabela 2), apresentaram menor área e foram mais espessas. No dossel superior, além da alta densidade de fluxo de fótons, outros fatores, como temperaturas elevadas e maiores déficits de pressão de vapor d'água (LÜTTGE, 1997) podem intervir nas características morfofisiológicas foliares. No entanto, além do efeito ocasionado pelo microclima do dossel superior, cabe ressaltar que a espécie em estudo ocorre preferencialmente na parte baixa da floresta, sujeita à seca sazonal. Nas folhas menores, a camada adjacente entre a folha e a atmosfera é reduzida, favorecendo, assim, a perda de calor por convecção e auxiliando a manutenção de temperaturas foliares abaixo das temperaturas do ar (LAMBERS et al., 1998). Entretanto, os folíolos de indivíduos jovens expostos a baixas densidades de fluxo de fótons foram mais finos e exibiram áreas maiores em relação aos indivíduos adultos, características essas que podem contribuir para o aumento da absorção de luz em um ambiente onde esse recurso é limitante (POORTER et al., 2000).

A relação entre a massa da folha e a sua área, a massa específica da folha, têm sido amplamente utilizada como parâmetro indicador da adaptação das plantas à diferentes condições de ambiente (BOEGER e WISNIEWSKI, 2003).Folhas dos indivíduos adultos apresentaram maiores valores de massa específica foliar, e esses valores foram semelhantes àqueles encontrados por outros autores para espécies arbóreas em dosséis de florestas tropicais úmidas (SANTIAGO et al., 2004). O aumento da massa foliar específica foi ocasionado pela maior espessura das folhas. Juntamente com a maior proporção do parênquima paliçádico, é provável que tais folhas apresentem maiores taxas fotossintéticas, em razão da maior proporção de células fotossintetizantes por unidade de área e do aumento da quantidade de enzimas fotossintéticas, que é observado em folhas cujo mesofilo é bem desenvolvido (EVANS e POORTER, 2001). Embora neste estudo as folhas estivessem expostas a diferentes regimes de luz, é possível que mudanças ontogenéticas possam estar envolvidas (ISHIDA et al., 2005). Alguns estudos têm mostrado que folhas de indivíduos adultos que se desenvolvem no dossel superior apresentam maiores valores de massa específica em relação aos jovens, quer estes estejam crescendo sob ambiente de sombra ou de sol (RIJKERS et al., 2000; THOMAS e WINNER, 2002).

A menor massa específica das folhas nos indivíduos jovens foi ocasionada, provavelmente, pela menor espessura foliar em comparação com aos indivíduos adultos. Fato semelhante foi observado por Aranda et al. (2004), trabalhando com oito espécies de clima temperado, em que o valor de $\mathrm{r}^{2}$ nas relações entre a espessura da lâmina foliar e a massa específica das folhas variou entre 0,40 e 0,97, para Ilex aquifolium L. (Aquifoliaceae) e Fagus sylvatica L. (Fagaceae), respectivamente.

R. Árvore, Viçosa-MG, v.33, n.5, p.885-893, 2009 
Nas folhas que se desenvolveram em condições de sombra, a diminuição das camadas de parênquima paliçádico e da espessura foliar pode favorecer maior intercepção da luz e maior capacidade de difusão de $\mathrm{CO}_{2}$ atmosférico nos sítios de carboxilação nos cloroplastos (BJÖRKMAN, 1981; WANG et al., 1994), contribuindo para a manutenção de um balanço positivo de carbono (KITAJIMA, 1994). Entretanto, analisando os dados da Tabela 3, observou-se que folhas de indivíduos jovens apresentaram menores razões de massa seca dos folíolos e maiores razões da massa seca da ráquis quando comparadas com as folhas dos indivíduos adultos. Tais resultados apontam para uma alocação ao tecido de suporte em detrimento do tecido fotossintético, o que não seria esperado para plantas que se regeneram em ambiente limitado pela disponibilidade de radiação luminosa, como C. echinata.

Em suma, C. echinata exibiu características que aparentemente permitem maior absorção de luz em ambiente sombreado, e ao mesmo tempo os dados indicam maior alocação de carbono para o suporte. Essas observações estão de acordo com o seu estádio sucessional e com a sua estratégia de ocupação dos espaços gerados pela formação de pequenas clareiras em ambiente de floresta semidecídua. Vale, ainda, salientar que diferenças nas características morfofisiológicas foliares foram observadas entre os indivíduos jovens, as quais podem estar relacionadas à heterogeneidade de distribuição de radiação luminosa no interior da floresta (MONTGOMERY e CHAZDON, 2002), bem como à variabilidade genética da população, uma vez que a espécie em estudo são plantas alógamas. Tendo em vista que a sobrevivência e o crescimento de indivíduos jovens de $C$. echinata estão sendo promovidos na ausência de grandes clareiras, a dinâmica estacional de formação de pequenas e grandes clareiras, assim como a distribuição espacial de fachos de luz no interior da floresta, são questões que merecem destaque em estudos futuros.

\section{AGRADECIMENTOS}

Os autores que participaram do III Curso de Campo de Ecologia de Dossel agradecem aos organizadores do curso, Profa. Talita Fontoura e Prof. Sérvio Pontes Ribeiro; aos proprietários da Reserva Particular do Patrimônio Natural RPPN Serra do
Teimoso Henrique Berbert e Lucélia Berbert; aos financiadores do curso (Global Canopy Programme International Canopy Network, Fundação Boticário de Proteção a Natureza, Universidade Estadual de Santa Cruz, Universidade Federal de Ouro Preto, Universidade Estadual de Campinas, Embaixada Britânica FCO, Aliança da Mata Atlântica, Ministério do Meio Ambiente Secretaria de Biodiversidade e Florestas e Instituto de Estudos Socioambientais do Sul da Bahia); ao Centro Universitário de Caratinga, pelo financiamento do translado do autor Antonio José Dias Vieira; e aos monitores Marcia Rocca e Wesley Duarte da Rocha (Spixo) e aos escaladores Ivan Soler, Marcial C. Jorge, Tilson Silva Nascimento, Luiz Eduardo Azevedo Rocha e Geraldo Santos Adriano (Canela),pela grande ajuda. Maria Cristina Sanches é grata à FAPEMIG (CRA 00099/04), pela bolsa Pósdoc júnior concedida a ela.

\section{REFERÊNCIAS}

AGUIAR, F. F. A. et al. Germinação de sementes e formação de mudas de Caesalpinia echinata Lam. (Pau-brasil): efeito de sombreamento. Revista Árvore, v.29, n.6, p.871-875, 2005.

AMORIM, A. M. et al. The vascular plants of a forest fragment in southern Bahia, Brazil. SIDA, v.21, p.1726-1752, 2005.

APHALO, P. J.; BALLARÉ, C. L. On the importance of information -acquiring systems in plant-plant interactions. Functional Ecology, v.9, n.1, p.5-14, 1995.

ARANDA, I. et al. Anatomical basis of the change in leaf mass per area and nitrogen investiment with relative irradiance within the canopy of eight temperate tree species. Acta Oecologica, v.25, n.3, p.187-195, 2004.

BJÖRKMAN, O. Responses to different quantum flux densities. In: LANGE, O.L.; OSMOND, C. B. \& ZIEGLER, H., (Eds.). Physiological plant ecology I. Encyclopedia of Plant Physiology. Heidelberg: Springer, 1981. v.12. p.57-107.

BOEGER, M. R. T.; WISNIEWSKI, C. Comparação da morfologia foliar de espécies arbóreas de três estádios sucessionais distintos de floresta ombrófila densa (Floresta Atlântica) no sul do Brasil. Revista Brasileira de Botânica, v.26, n.1, p.61-72, 2003. 
CARVALHO, P. E. R. Espécies florestais brasileiras. Brasília, Embrapa-SPI, 1994.

CORRÊA, A. M. S. Morfologia polínica de Caesalpinia echinata Lam. Revista Brasileira de Botânica, v.26, n.3, p.355-359, 2003.

DUZ, S. R. et al. Crescimento inicial de três espécies arbóreas da Floresta Atlântica em resposta à variação na quantidade de luz. Revista Brasileira de Botânica, v.27, n.3, p.587-596, 2004.

EVANS, J. R.; POORTER, H. Photosynthetic acclimation of plants to growth irradiance: the relative importance of specific leaf area and nitrogen partitioning in maximizing carbon gain. Plant, Cell and Environment, v.24, n.8, p.755-767, 2001.

GEbLER, A. et al. Ecophysiology of selected tree species in different plant communities at the periphery of the Atlantic Forest of SE - Brazil III. Three legume trees in a semi-deciduous dry forest. Trees,v.19, n.5, p.523-530, 2005.

ISHIDA, A.; YAZAKI, K.; HOE, A. L. Ontogenetic transition of leaf physiology and anatomy from seedlings to mature trees of a rain forest pioneer tree, Macaranga gigantea. Tree Physiology, v.25, n.5, p.513-522, 2005.

KITAJIMA, K. Relative importance of photosynthetic traits and allocation patterns as correlates of seedling shade tolerance of 13 tropical tree species. Oecologia,v.98, NUMERO, p.419-428, 1994.

LAMBERS, H.; CHAPIN III, S. T.; PONS, T. J. Plant physiological ecology. New York: Springer-Verlag, 1998.

LEE, D. W. et al.. Effects of irradiance and spectral quality on seedling development of two southeast Asian Hopea species. Oecologia, v.110, n.1, p.1-9, 1997.

LIMA JUNIOR, E. C. et al. Aspectos fisioanatômicos de plantas jovens de Cupania vernalis Camb. submetidas a diferentes níveis de sombreamento. Revista Árvore, v.30, n.1, p.33-46, 2006.
LÜtTGE, U. Physiological ecology of tropical plants. Berlin: Springer, 1997.

MELO, S. C. O. et al. Microsatellite markers for Caesalpinia echinata Lam. (Brazilwood), a tree that named a country. Conservation Genetics, v.8, n.6, p.1269-1271, 2007.

MONTGOMERY, R. A.; CHAZDON, R. L. Light gradient partitioning by tropical tree seedlings in the absence of canopy gaps. Oecologia, v.131, n.1, p.165-174, 2002.

POORTER, L. et al. Leaf optical properties in Venezuelan cloud forest trees. Tree Physiology, v.20, n.8, p.519-526, 2000.

REZENDE, C. M. et al. Constituintes químicos voláteis das flores e folhas do pau-brasil (Caesalpinia echinata Lam.). Química Nova, v.27, n.3, p.414-416, 2004.

RIJKERS, T.; PONS T. L.; BONGERS, F. The effect of tree height and light availability on photosynthetic leaf traits of four neotropical species differing in shade tolerance.

Functional Ecology, v.14, n.1, p.77-86, 2000.

SANTIAGO, L. S. et al. Coordinated changes in photosynthesis, water relations and nutritional traits of canopy trees along a precipitation gradient in lowland tropical forest.

Oecologia, v.139, n.4, p.495-502, 2004

THOMAS, S. C.; WINNER W. E. Photosynthetic differences between saplings and adult trees: an integration of field results by meta-analysis.

Tree Physiology,v.22, n.2-3, p.117-127, 2002.

WANG, G. G.; QIAN, H.; KLINKA, K. Growth of Thuja plicata seedlings along a light gradient. Canadian Journal of Botany, v.72, n.12, p.1749-1757, 1994. 
